\title{
Dynamic methods of spatial calculation of structures based on a plate model
}

\author{
Elyor Toshmatov ${ }^{1, *}$, Makhamtali Usarov ${ }^{2}$, Gayratjon Ayubov ${ }^{2}$, Davronbek Usarov $^{2}$ \\ ${ }^{1}$ Department of Theoretical and Constructional Mechanics, Tashkent Institute of Irrigation and \\ Agricultural Mechanization Engineers, 39 KoriNiyoziy str., Tashkent 100000, Uzbekistan \\ ${ }^{2}$ Institute of Mechanics and Seismic Stability of Structures of the Academy of Sciences of the \\ Republic of Uzbekistan, 31 “Do'rmon yo'li” str., Tashkent 100125, Uzbekistan
}

\begin{abstract}
This article was devoted to the development of methods of the dynamic calculation based on the finite difference method of laminar structures in the framework of the bimoment theory, which takes into account the spatial stress-strain state. Were given the solutions of the problem of transverse vibrations of the plate model of structures.
\end{abstract}

\section{Introduction}

Structures such as water retaining dams, dykes, water reservoirs, etc., built and operated in seismically active regions of the Republic of Uzbekistan, are subjected to loads of both static (gravitational forces, natural external loads, etc.) and dynamic (seismic) nature. In dynamic calculation of this type of structures according to the "standard procedure, designers are limited by the possibility of obtaining only the most approximate reliability estimates.The ways to the main reserves of increasing the efficiency of the structureconstructions are unavailable, since these reserves are found only when considering the wave nature of dynamic loads (seismic) and are connected with the necessity of assuming irreversible strains in a structure". To determine the reserve capabilities of water retaining structures in calculations, various linear and nonlinear models are used in a planeor spatial form [1-7].

This article proposes a method for calculating the structures for seismic resistance on the basis of a continuum plate model developed in the framework of the bimoment theory [8-17], taking into account the spatial stress-strain state. If to consider the law of nonlinearity of displacements distribution in the cross-sections of the plate, then in addition to tensile and shear forces, bending and torsional moments, there appear the additional force factors, called the bimoments.

\section{Method}

In the development and solution of the problem of bending and vibrations of thick plates is based on bimoment theory of plates built within the three-dimensional theory of elasticity

\footnotetext{
* Corresponding author: toshmatov.elyor@bk.ru
} 
without simplifying hypotheses, using the method of displacements expansion into Maclaurin infinite series on one of the spatial coordinates.

It is assumed that the displacement of the structure base occurs only along the horizontal transverse direction according to the following law:

$$
u_{1}=u_{2}=0, \quad u_{3}=u_{30}(t) .
$$

Where $u_{30}(t)$ is the law of base motion, given in the following form:

$$
u_{30}(t)=A_{30} \sin \left(\omega_{0} t\right)
$$

where $A_{0}$ and $\omega_{0}$ are the amplitude (maximum value) and frequency of displacements of the base $\omega_{0}=\frac{2 \pi}{T_{0}}$. In calculations we take $A_{0}=3 \mathrm{~mm}$ and $T_{0}=0.1 \mathrm{~s}$.

The plate is under water pressure distributed along the vertical according to a law:

$$
p=p_{0}(b-y),
$$

where $p_{0}$ is the pressure parameter, $b$ is the height of the dam.

As the equations of motion of the plate model of a structure, the equations of bendingshear oscillations of a thick orthotropic plate are taken, which are written relative to bending, torsional moments, shearing forces, bimoments and kinematic equations in the form:

$$
\begin{gathered}
\frac{\partial M_{11}}{\partial x_{1}}+\frac{\partial M_{12}}{\partial x_{2}}-Q_{13}+H \tilde{q}_{1}=\frac{H^{2}}{2} \rho \ddot{\tilde{\psi}}_{1}, \quad \frac{\partial M_{21}}{\partial x_{1}}+\frac{\partial M_{22}}{\partial x_{2}}-Q_{23}+H \tilde{q}_{2}=\frac{H^{2}}{2} \rho \ddot{\tilde{\Psi}}_{2}, \\
\frac{\partial P_{11}}{\partial x_{1}}+\frac{\partial P_{12}}{\partial x_{2}}-3 \tilde{p}_{13}+H \tilde{q}_{1}=\frac{H^{2}}{2} \rho \ddot{\tilde{\beta}}_{1}, \quad \frac{\partial P_{21}}{\partial x_{1}}+\frac{\partial P_{22}}{\partial x_{2}}-3 \tilde{p}_{23}+H \tilde{q}_{2}=\frac{H^{2}}{2} \rho \ddot{\tilde{\beta}}_{2} \\
\frac{\partial Q_{13}}{\partial x_{1}}+\frac{\partial Q_{23}}{\partial x_{2}}+2 \tilde{q}_{3}=\rho H \ddot{\tilde{r}} \\
H \frac{\partial \tilde{p}_{13}}{\partial x_{1}}+H \frac{\partial \tilde{p}_{23}}{\partial x_{2}}-4 \tilde{p}_{33}+2 \tilde{q}_{3}=H \rho \tilde{\tilde{\gamma}} \\
\tilde{u}_{1}=\frac{1}{2}\left(21 \tilde{\beta}_{1}-7 \tilde{\psi}_{1}\right)-\frac{1}{30} H \frac{\partial \tilde{W}}{\partial x_{1}}+\frac{1}{30} \frac{H \tilde{q}_{1}}{G_{13}} \\
\tilde{u}_{2}=\frac{1}{2}\left(21 \tilde{\beta}_{2}-7 \tilde{\psi}_{2}\right)-\frac{1}{30} H \frac{\partial \tilde{W}}{\partial x_{2}}+\frac{1}{30} \frac{H \tilde{q}_{2}}{G_{23}} \\
\tilde{W}=\frac{1}{4}(21 \tilde{\gamma}-3 \tilde{r})-\frac{1}{20} H\left(\frac{E_{31}}{E_{33}} \frac{\partial \tilde{u}_{1}}{\partial x_{1}}+\frac{E_{32}}{E_{33}} \frac{\partial \tilde{u}_{2}}{\partial x_{2}}\right)+\frac{H \tilde{q}_{3}}{20 E_{33}} .
\end{gathered}
$$




\section{Results}

\subsection{Analysis of numerical results for a plate model of structures (dams)}

The calculations have been conducted for an isotropic plate with plate dimensions in plan $\mathrm{a}=25 \mathrm{~m}, \mathrm{~b}=25 \mathrm{~m}, \mathrm{H}=8 \mathrm{~m}$.

From kinematic conditions (1) and (2) it follows that in the sidel faces and in the base of the dam $x_{1}=0, x_{2}=a$ and $x_{2}=0$, the boundary conditions must be met in the form:

$$
\tilde{\psi}_{1}=0, \tilde{\psi}_{2}=0, \tilde{\beta}_{1}=0, \tilde{\beta}_{2}=0, \tilde{u}_{1}=0, \tilde{u}_{2}=0, \quad \tilde{r}=u_{30}(t), \tilde{\gamma}=\frac{1}{3} u_{30}(t), \quad \tilde{W}=u_{30}(t)
$$

At the free upper face of the dam construction, the conditions for zero force factors are obtained

$$
\begin{aligned}
& M_{12}=0, M_{22}=0, P_{12}=0, P_{22}=0, Q_{23}=0, \quad \tilde{p}_{23}=0 \text {, } \\
& \tilde{p}_{23}=0, \quad \tilde{\sigma}_{11}=0, \quad \tilde{\sigma}_{12}=0, \quad \sigma_{22}^{*}=0
\end{aligned}
$$

Figures 1-5 show the modes of oscillation, the law of change in time of displacements and force factors of the plate - dam.

Figure 1 shows the graphs for changing the dimensionless values of the deflection $r$ of the plate depending on dimensionless time $\tau$. From the graph it is seen that at the point of the plate $x_{1}=\frac{a}{2}, y_{1}=b$, the dimensionless value of the deflection, reaching the maximum value, is $r=2.526$ (figure $1, b$ ).

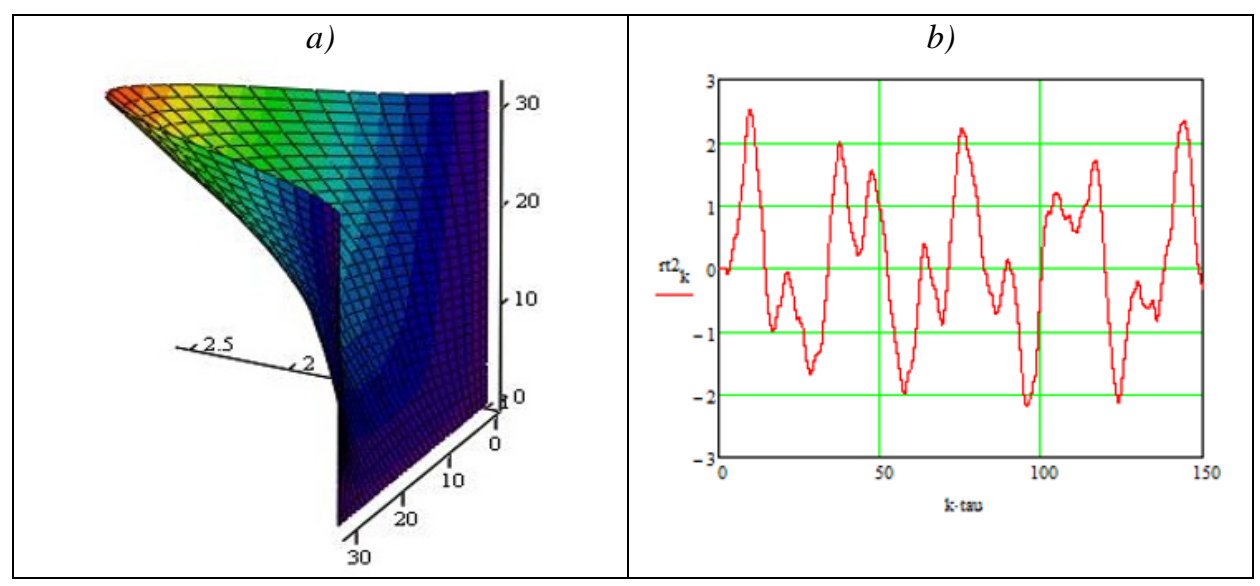

Fig.1. Modes of oscillations of the plate structure (dam) (a) and the graph of deflection $r$ changes

$$
\text { over time (b) }
$$

Figure 2 shows the stress $\sigma_{11}$ isolines. Figure 3 shows the graphs of changes in the dimensionless values of the stress $\sigma_{11}$ of the plate depending on the dimensionless time $\tau$. It is clear from the graph that at the point of the plate $x_{1}=0, y_{1}=b$, the dimensionless stress value reaching the maximum value is $\sigma_{11}=-1,574$ (figure 3 ). 


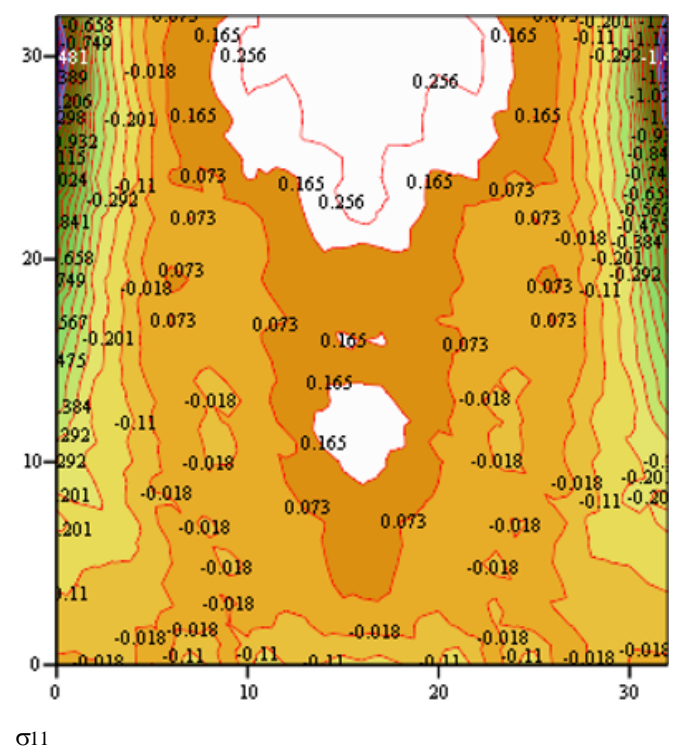

Fig.2. Isolines of stress $\sigma_{11}$.

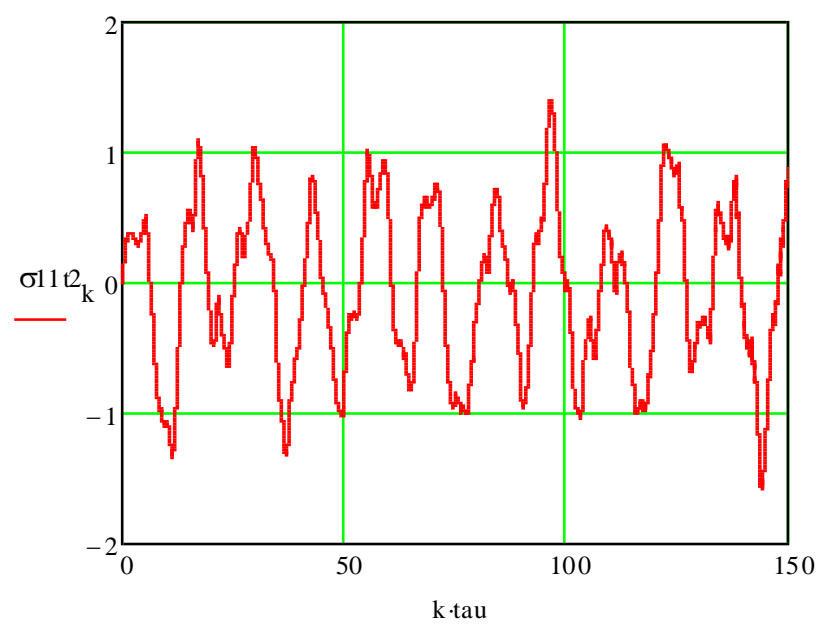

Fig.3. Graphs of stress $\sigma_{11}$ changes over time

Figure 4 shows the isolines of stress $\sigma_{22}$. Figure 5 shows the graphs of changes in the dimensionless values of stress $\sigma_{22}$ of the plate depending on the dimensionless time $\tau$. It is clear from the graph that at the point of the plate $x_{1}=\frac{a}{2}, y_{1}=0$, the dimensionless stress value reaching the value $\sigma_{22}=-0.772$ (figure 5) is maximal. 


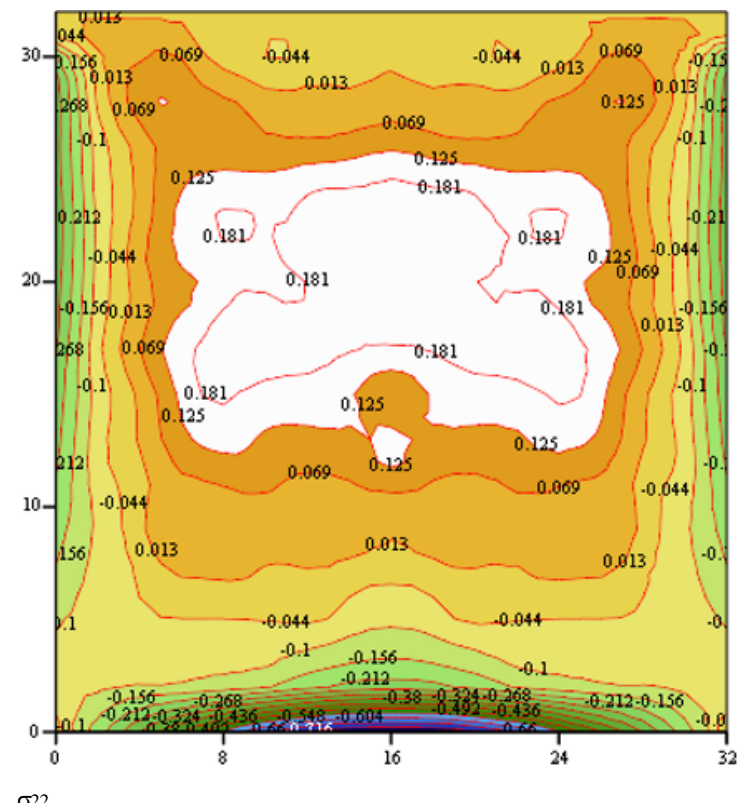

Fig.4. Isolines of stress $\sigma_{22}$.

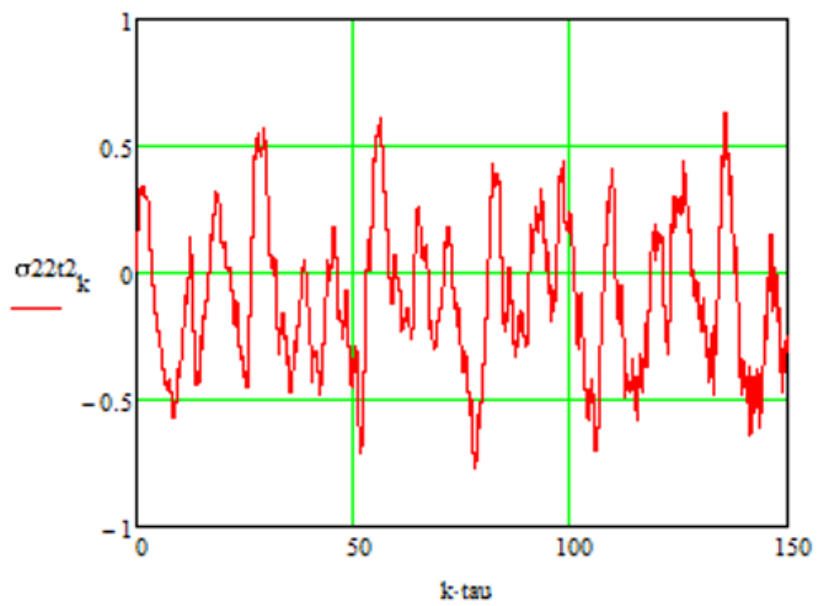

Fig.5. Graphs of stress change $\sigma_{22}$ over time.

Table 1, a. Maximum dimensionless values of displacement, stress, moments and shear forces on a plate - dam

\begin{tabular}{|l|c|c|c|c|c|c|c|}
\hline \multicolumn{1}{|c|}{ State } & $\tilde{r} / A_{0}$ & $\frac{\tilde{\sigma}_{11} H}{E_{1} A_{0}}$ & $\frac{\tilde{\sigma}_{22} H}{E_{1} A_{0}}$ & $\frac{\tilde{M}_{11}}{E_{1} H A_{0}}$ & $\frac{\tilde{M}_{22}}{E_{1} H A_{0}}$ & $\frac{\tilde{Q}_{13}}{E_{1} A_{0}}$ & $\frac{\tilde{Q}_{23}}{E_{1} A_{0}}$ \\
\hline With water & 2.526 & -1.574 & -0.772 & -0.156 & -0.087 & 0.448 & 0.242 \\
\hline $\begin{array}{l}\text { Without } \\
\text { water }\end{array}$ & 2.435 & -1.523 & -0.668 & -0.151 & -0.077 & 0.440 & 0.211 \\
\hline
\end{tabular}


Table 1,b. Maximum dimensional values of displacement, stress, moments and shear forces on the plate - dam

\begin{tabular}{|l|c|c|c|c|c|c|c|}
\hline \multicolumn{1}{|c|}{ State } & $\begin{array}{c}r, \\
\left(\mathbf{1 0} \mathbf{- n}^{-3} \mathbf{m}\right)\end{array}$ & $\begin{array}{c}\sigma_{11}, \\
(\mathbf{M P a})\end{array}$ & $\begin{array}{c}\sigma_{22}, \\
(\mathbf{M P a})\end{array}$ & $\begin{array}{c}M_{11}, \\
\left(\mathbf{M P a} \cdot \mathbf{m}^{2}\right)\end{array}$ & $\begin{array}{c}M_{22}, \\
\left(\mathbf{M P a} \cdot \mathbf{m}^{2}\right)\end{array}$ & $\begin{array}{c}Q_{13}, \\
(\mathbf{M P a} \cdot \mathbf{m})\end{array}$ & $\begin{array}{c}Q_{23}, \\
(\mathbf{M P a} \cdot \mathbf{m})\end{array}$ \\
\hline $\begin{array}{l}\text { With } \\
\text { water }\end{array}$ & 7.578 & -11.805 & -5.790 & -74.880 & -41.760 & 26.880 & 14.520 \\
\hline $\begin{array}{l}\text { Without } \\
\text { water }\end{array}$ & 7.305 & -11.422 & -5.010 & -72.480 & -36.960 & 26.400 & 12.660 \\
\hline
\end{tabular}

In calculations, the calculation step for the dimensionless coordinates is taken as $\Delta x=\Delta y=\frac{1}{32}$. The stability of iteration over dimensionless time is provided by an explicit scheme with a step $\Delta \tau=0.01$. Here $x=\frac{x_{1}}{a}, y=\frac{x_{2}}{b}, \tau=\frac{c t}{H}$.

\subsection{Analysis of numerical results for a orthotrop plate model of structures (wall)}

As equations of motion of aorthotrop plate model of structure, the equations of bendingshear oscillations is taken, which are written relative to the bending, torque, cutting forces, bimoments, and kinematic equations in the form. At the base of the structure, the boundary conditions for the bending-shear oscillations have the form:

$$
\tilde{\psi}_{1}=0, \tilde{\psi}_{2}=0, \tilde{\beta}_{1}=0, \tilde{\beta}_{2}=0, \quad \tilde{u}_{1}=0, \tilde{u}_{2}=0, \quad \tilde{r}=u_{30}(t), \tilde{\gamma}=\frac{1}{3} u_{30}(t), \quad \tilde{W}=u_{30}(t) \text {. }
$$

On the free face of the structure, the conditions for zero force factors are

$$
\begin{gathered}
M_{11}=0, M_{12}=0, P_{11}=0, P_{12}=0, Q_{13}=0, \\
\tilde{p}_{13}=0, \quad \tilde{\sigma}_{11}=0, \quad \tilde{\sigma}_{12}=0, \quad \sigma_{11}^{*}=0 .
\end{gathered}
$$

On the free upper face of the structure the conditions are

$$
\begin{aligned}
& M_{12}=0, M_{22}=0, \quad P_{12}=0, P_{22}=0, Q_{23}=0, \\
& \tilde{p}_{23}=0, \quad \tilde{\sigma}_{11}=0, \quad \tilde{\sigma}_{12}=0, \quad \sigma_{22}^{*}=0 .
\end{aligned}
$$

Figures 6 and 7 show the modes of oscillation, the law of change in time of displacements and force factors of the wall.

Figure 6 shows the graphs for changing the dimensionless values of the deflection $r$ of the wall depending on dimensionless time $\tau$. From the graph it is seen that at the point of the wall $x_{1}=a, y_{1}=b$, the dimensionless value of the deflection, reaching the maximum value, is $r=1.52$ (figure $1, b$ ). 
a)

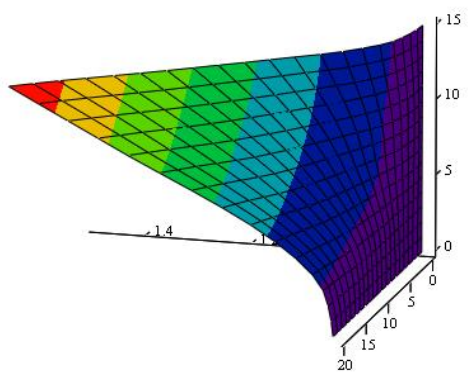

b)

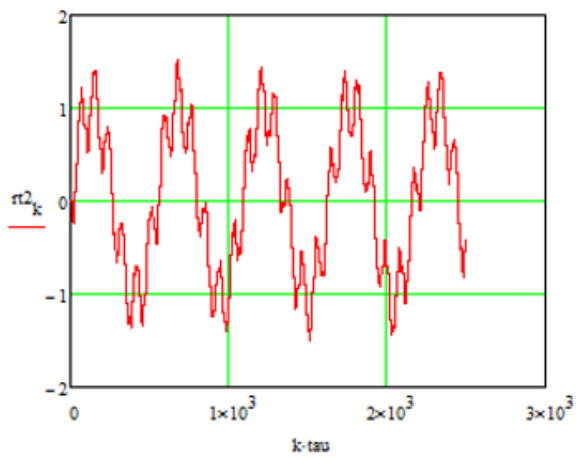

Fig.6. Modes of oscillations of theplate structure (wall) (a) and the graph of deflection $r$ changesover time (b)

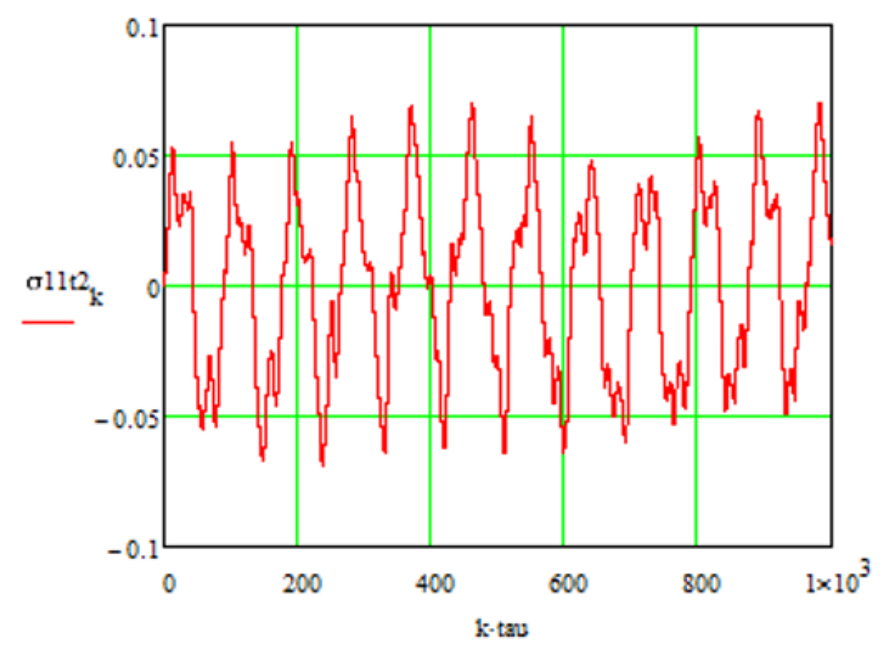

Fig.7. Graphs of stress $\sigma_{11}$ changes over time

Figure 7 shows the graphs of changes in the dimensionless values of the stress $\sigma_{11}$ of the wall depending on the dimensionless time $\tau$. It is clear from the graph that at the point of the wall $x_{1}=0, y_{1}=b$, the dimensionless stress value reaching the maximum value is $\sigma_{11}=0,07$ (figure 7).

Table 2. Maximum dimensionless values of displacement, stress, moments and shear forces on the plate-wall

\begin{tabular}{|l|c|c|c|c|c|c|c|}
\hline $\begin{array}{c}\text { Dimensions } \\
\text { of the plate } \\
\text { - wall (m) }\end{array}$ & $\tilde{r} / A_{0}$ & $\frac{\tilde{\sigma}_{11} H}{E_{1} A_{0}}$ & $\frac{\tilde{\sigma}_{22} H}{E_{1} A_{0}}$ & $\frac{\tilde{M}_{11}}{E_{1} H A_{0}}$ & $\frac{\tilde{M}_{22}}{E_{1} H A_{0}}$ & $\frac{\tilde{Q}_{13}}{E_{1} A_{0}}$ & $\frac{\tilde{Q}_{23}}{E_{1} A_{0}}$ \\
\hline $4 \times 3 \times 0.5$ & 1.52 & 0.07 & -0.051 & 0.013 & 0.01 & 0.00938 & 0.011 \\
\hline
\end{tabular}


In calculations, the calculation step for the dimensionless coordinates is taken as $\Delta x=\frac{1}{20}, \Delta y=\frac{1}{15}$. The stability of the iteration on a dimensionless time is provided by an explicit scheme at step $\Delta \tau=0.01$. Here $x=\frac{x_{1}}{a}, y=\frac{x_{2}}{b}, \tau=\frac{c t}{H}$.

\section{Conclusion}

Bimoment theory is a new theory which is built to calculate the precise displacements, forces, moments, bimoments and stresses of the plate, and it is applied to solving the dynamic problem of forced oscillations of orthotropic thick plate and it's based on the method of finite differences.

In conclusion, analysis of the obtained results shows that the developed methods for numerical solving seismic oscillation problems and the method, algorithm and dynamic calculation program based on the finite difference method of plate structures in the framework of the bimomental theory adequately fully reflect the stress-strain state of plate structures with seismic effects with and without water pressure..

\section{References}

1. M.M. Mirsaidov, T.Z. Sultanov. Soil Mechanics and Foundation Engineering, 49(6) (2013)

2. M.M. Mirsaidov, T.Z. Sultanov, D.F. Rumi. Magazine of Civil Engineering, 39(4) (2013)

3. T.Z. Sultanov, D.A. Khodjaev, M.M. Mirsaidov. Magazine of Civil Engineering, 45(1) (2014)

4. M.V. Sukhoterin, S.O. Baryshnikov, T.P. Knysh, R.A. Abdikarimov. Magazine of Civil Engineering, 6(82) (2018)

5. M.M. Mirsaidov, T.Z. Sultanov, R.A. Abdikarimov, A.N. Ishmatov, B.Sh. Yuldoshev, E.S. Toshmatov, D.P. Jurayev. Magazine of Civil Engineering, 6(82) (2018)

6. M.M.Mirsaidov, R.A.Abdikarimov, N.I.Vatin, V.M.Zhgutov, D.A.Khodzhaev, B.A.Normuminov. Magazine of Civil Engineering, 1(77) (2018)

7. M.V. Sukhoterin, S.O. Baryshnikov, T.P. Knysh. Magazine of Civil Engineering, 8(76) (2017)

8. M.K. Usarov, D.M. Usarov. Scientific Bulletin of SamSU, 3(109) (2018)

9. M.K. Usarov. Magazine of Civil Engineering, 1(53) (2015)

10. M.K. Usarov. Magazine of Civil Engineering, 4(22) (2011)

11. M.K. Usarov, G.T. Ayubov. Uzbek magazine "Problems of Mechanics", Tashkent, 1 (2018)

12. M.K. Usarov, G.T. Ayubov. Journal of Applied Mathematics and Physics, 6(9) (2018)

13. M.K. Usarov. J.Applied Mathematics and Physics, 5(10) (2017)

14. M.K. Usarov. World Journal of Mechanics, 6 (2016.)

15. M.K. Usarov, D.M. Usarov, G.T. Ayubov. Journal of Applied Mathematics and Physics, 4 (2016)

16. M.K. Usarov. Open Journal of Applied Sciences, 5 (2016)

17. M.Mirsaidov, I.E.Troyanovskii. Forced axisymmetric oscillations of a viscoelastic cylindrical shell. Polymer Mechanics, 1975. 11(6), Pp. 953-955 